\title{
High Efficiency White Light-Emitting Device Based on Quantum Dots/Polymer Hybrid Emissive Layer
}

\author{
Sang-Hyup Kim ${ }^{1}$, Jun-Woo Lee ${ }^{1}$, Jae-Sung Lee ${ }^{1}$, Sang-Won Lee ${ }^{1}$, Sae-Wan Kim ${ }^{1}$, Ju-Seong Kim ${ }^{1}$, Kyung-Jae Choi ${ }^{2}$, \\ Jung-Hee Lee ${ }^{1}$, Dae-Hyuk Kwon ${ }^{3}$ and Shin-Won Kang ${ }^{1, *}$ \\ ${ }^{1}$ School of Electronics Engineering, College of IT Engineering, Kyungpook National University, 80 Daehakro,Bukgu, 41566 \\ Daegu, Republic of Korea \\ ${ }^{2}$ Department of Sensor and Display Engineering, Kyungpook National University, 80 Daehakro, Bukgu,41566 Daegu, Republic of \\ Korea \\ ${ }^{3}$ Department of Electronic Engineering, Kyungil University, 50 Gamasil-Gil, Hayang-Eup, Gyeongbuk,38428 Gyeongsan-Si, \\ Republic of Korea \\ *Corresponding author
}

\begin{abstract}
We propose all-solution processed white quantum dots light emitting devices (WQLEDs) based on CdSe/ZnS Quantum dots(QDs)/polymer hybrid emissive layer which was red, green and blue (RGB) QDs and blue emitting homo-polymer blending. The proposed WQLED showed higher brightness, external current efficiency and color purity than only RGB blended WQLEDs. The maximum luminance was $11,600 \mathrm{~cd} / \mathrm{m}^{2}$ and a maximum current efficiency was $1.29 \mathrm{~cd} / \mathrm{A}$ at $7 \mathrm{~V}$ bias and exhibited the Commission Internationale de I Enclairage (CIE) coordinates of $(0.34,0.36)$ near white light region.
\end{abstract}

Keywords-component; quantum dots; white light-emitting devices; blue emitting homo-polymers

\section{INTRODUCTION}

White quantum dots (QDs) light emitting devices (WQLEDs) have been attracting much attention in the fast few years. Nowadays, many researchers have reported the generation of the white LEDs such as a tandem-type white LED using organic materials [1], QDs/polymers hybrid WQLED [2], and integration of red, green, and blue (RGB) QDs based WQLEDs. Among these applications, QDs/polymers hybrid type is a most simple structure to realize the WQLEDs. The white emission was achieved by tuning the concentration ratio of the QD/polymer blend.

Recently, QDs/polymers hybrid WQLEDs have attracted great interest due to the high emission efficiency. Park et al. proposed the WQLED by using the QDs/polymers nanocomposite into blue emitting poly(phenylene vinylene)polyfluorene copolymers [3]. Huang et al. the green and red QDs were incorporated into the polyfluorene (PFO) as the single emissive layer (EML) [4]. QDs/polymers hybrid structure enhanced brightness and efficiency due to efficient energy transfer from polymers to QDs [5]. However, reported hybrid WQDLEDs need complex process such as vacuum evaporation to deposit the charge transport layer.

In this study, in order to overcome complex process, we proposed solution-processible WQLED. Proposed WQDLED was composed $\mathrm{CdSe} / \mathrm{ZnS}$ quantum dots(QDs)/polymers hybridization, which was red, green and blue (RGB) QDs and blue emitting homo-polymer (ADS329BE) blending. In this structure, ADS329BE didn't role only hole buffer layer but also exciton energy transport to individual RGB QDs due to Förster energy transfer mechanism. Therefore, hybrid EML transfer exciton energy effectively and improve the performance of WQLED comparing to only RGB blended WQLED.

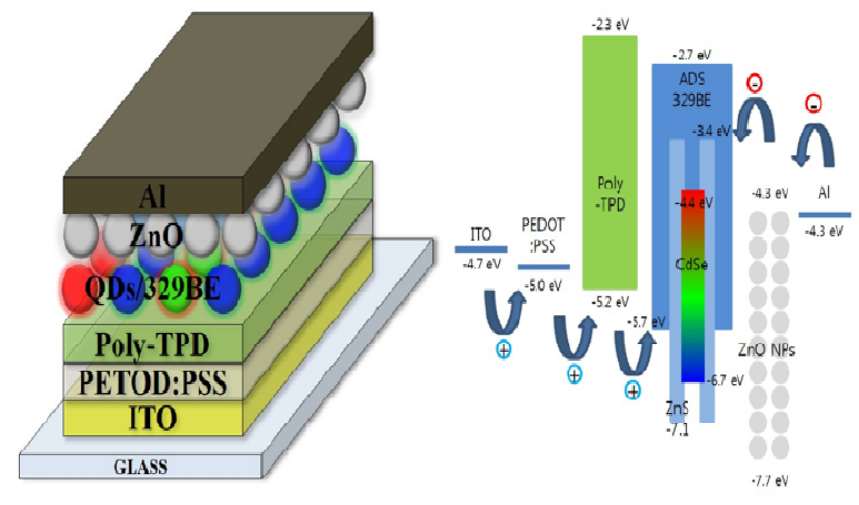

FIGURE I. SCHEMATIC AND ENERGY DIAGRAM OF WQLEDS

\section{EXPERIMENTAL SECTION}

The EML materials were dissolved in toluene at concentrations of $10 \mathrm{mg} / \mathrm{ml}$ for QDs and $0.5 \mathrm{wt} \%$ for ADS329BE, respectively. QD solutions for WQLEDs were prepared by mixing individual RGB QDs in volume ratio 1:1:4. In addition, ADS329BE was mixed with blended RGB QDs in volume ratio 1:1 for hybrid EML.

To fabricate WQLED, patterned ITO glass was sonicated in acetone, methanol, IPA and then dried by $\mathrm{N}_{2}$ gas blowing. The cleaned ITO glass was treated with UV ozone for $15 \mathrm{~min}$ to deposit a hydrophilic Poly (3, 4-eneioxythiophene): polystyrene sulfonate (PEDOT: PSS) onto hydrophobic surface of ITO glass. After UV ozone treatment, PEDOT:PSS used as a hole injection layer was deposited by spin coating at $3,000 \mathrm{rpm}$ for $30 \mathrm{~s}$, then annealed on $150^{\circ} \mathrm{C}$ hot plate for 10 min. Poly(N, N'-bis(4-butylphenyl-N,N'-bis(phenyl)benzidine (poly-TPD) as a hole transport layer and QDs/ADS329BE 
EML were annealed in vacuum oven at $110^{\circ} \mathrm{C}$ and $80^{\circ} \mathrm{C}$ for $30 \mathrm{~min}$, respectively, after spin coating process at 3,000 rpm during 60 s successively. ZnO nanoparticles (NPs) synthesized by sol-gel method were deposited onto EML with spin coating process at $1,500 \mathrm{rpm}, 30 \mathrm{~s}$. Finally, aluminum was thermally deposited with a thickness of $150 \mathrm{~nm}$ under high vacuum condition. Proposed device schematic and band diagram were shown in Figure 1. [6]

\section{RESUlts AND DisCUSSION}

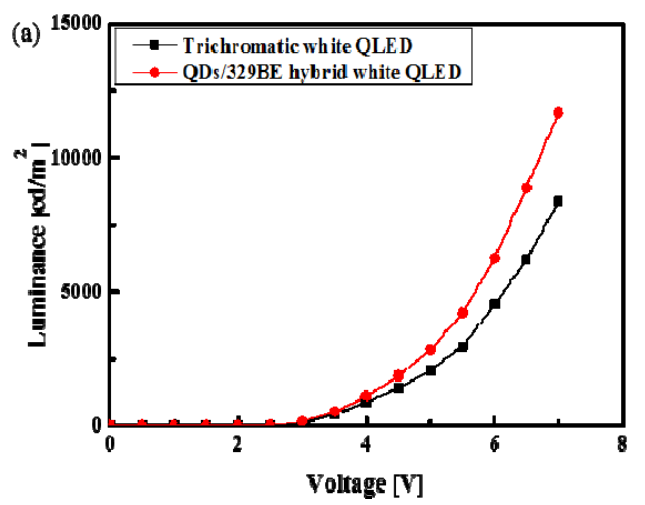

(b)

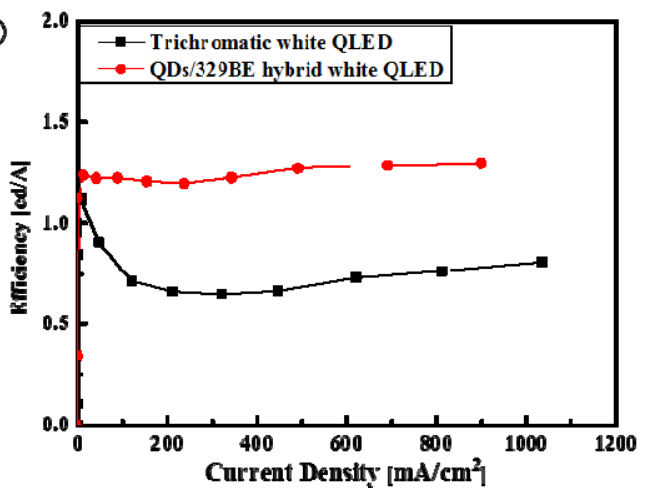

FIGURE II. CHARACTERISTICS OF WQLEDS ; (A) LUMINANCE OF WQLEDS, (B) CURRENT EFFICIENCY OF WQLEDS

We measured the I-V-L characteristics by computercontrolled voltmeter (Keithley 2400s) and luminance meter (CS100A). As it shown in Figure 2 (a) and (b), QDs/ADS329BE hybrid WQLED was highly improved than RGB blended trichromatic WQLED. Hybrid WQLED was measured near white coordinate of $(0.34,0.36)$ at $7 \mathrm{~V}$ with a luminance of $11,600 \mathrm{~cd} / \mathrm{m}^{2}$ and a current efficiency of 1.29 $\mathrm{cd} / \mathrm{A}$, whereas trichromatic WQLED was measured inferior brightness and current efficiency which were a luminance of $8400 \mathrm{~cd} / \mathrm{m}^{2}$ and $0.80 \mathrm{~cd} / \mathrm{A}$ at $7 \mathrm{~V}$ bias. CIE coordinates which are shown in Figure 3 also shifted to green region than hybrid WQLED such as $(0.32,0.44)$.

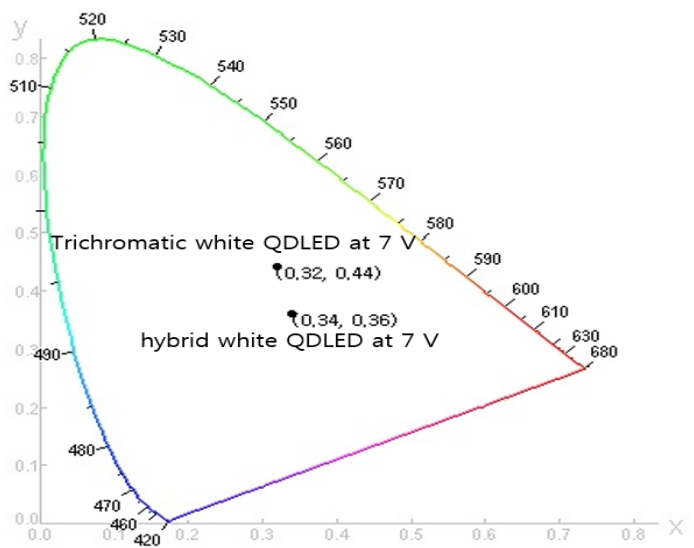

FIGURE III. CIE COORDINATE OF WQLEDS

According to the results, hybrid WQLED highly improved the performance than RGB trichromatic WQLED. We supposed that Förster energy transfer occurred effectively from 329BE to individual QDs in hybrid WQLED. To confirm the energy flow between 329BE and QDs, we measured absorbance spectrums of individual RGB QDs respectively via spectrometer (UV-1601), photoluminescence (PL) spectrum of 329BE via spectrometer (QE65000) and electroluminescence (EL) of fabricated WQLEDs via Mcscience I-V-L measure equipment (M6100 OLED I-V-L test system). Figure 4 show absorbance spectrums of RGB QDs and PL spectrum of 329BE. The PL spectrum of 329BE significantly overlap with RGB absorbance spectrum. The overlap demand for occurring efficient energy transfer from 329BE to QDs [5].

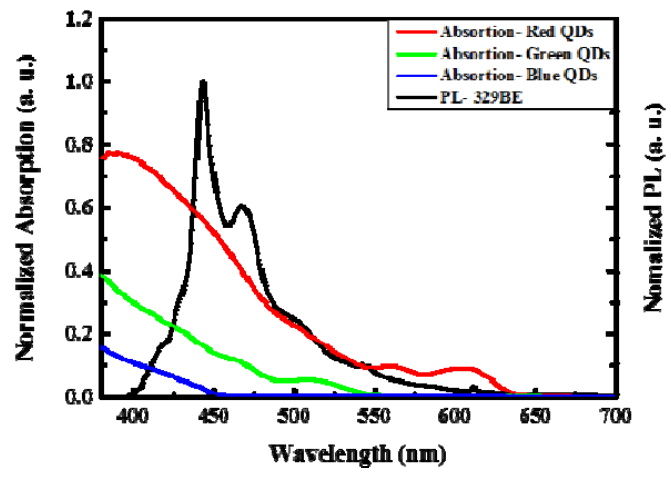

FIGURE IV. ABSORBANC OF RGB QDS AND PHOTO LUMINESCENCE OF 329BE

The EL spectrums of fabricated WQLEDs show the energy transition clearly. According to the Figure 5 (a), RGB trichromatic WQLED has very poor blue emission and that's why it couldn't emit white light. Besides, the EL spectrum of hybrid WQLED in Figure 5 (b) obviously show the increase of intensities of green EL as well as blue EL. As the EL spectrum of hybrid WQLED, most energy of 329BE had been transferred to QDs and the transition effected to improve the performance of hybrid WQLED. 

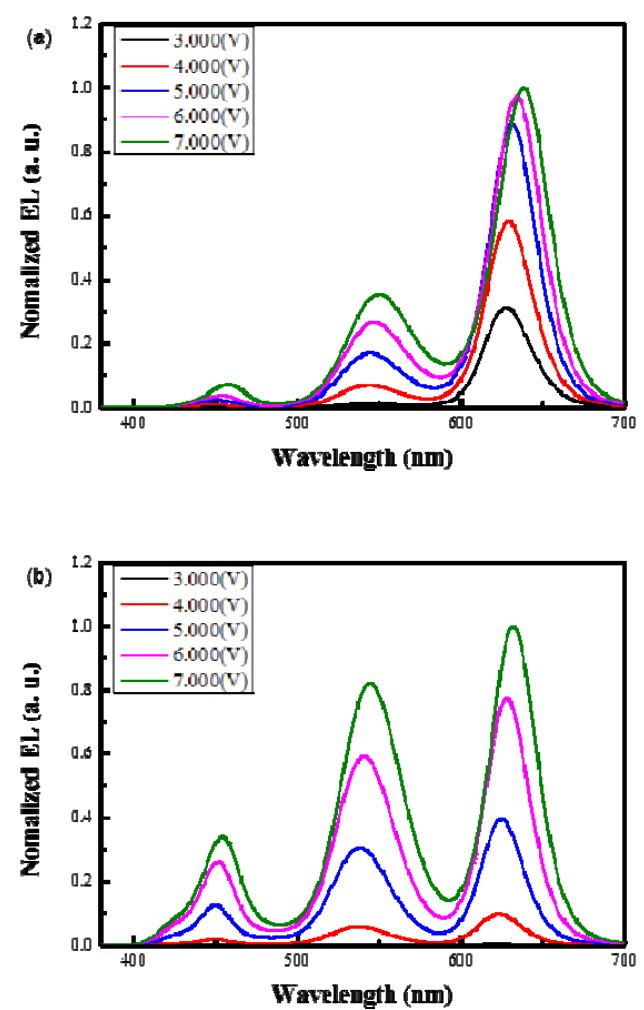

FIGURE V. ELECTRO LUMINESCENCE OF FABRICATED DEVICES; (A) RGB TRICHROMATIC WQLED, (B) HYBRID WQLED

\section{CONCLUSION}

In this paper, we analyzed the effect of QDs/ADS329BE hybrid EML and it is confirmed that hybrid EML improve the performance of WQLED significantly. The results indicate obviously that QDs/ADS329BE hybrid structure is simple and proper to fabricate high efficiency WQLEDs.

\section{ACKNOWLEDGEMENT}

This work was supported by Basic Science Research Program through the National Research Foundation of Korea (NRF) grant funded by the Korea government (MSIP) (No. NRF2014R1A2A1A11050377).

\section{REFERENCES}

[1] T. W. Lee, T. Noh, B. K. Choi, M. S Kim, and D. W. Shin, "Highefficiency stacked white organic light-emitting diodes," Appl. Phys. Lett, vol. 92 , p. 043301 , January 2008 .

[2] P. O. Anikeeva, J. E. Halpert, M. G. Bawendi, and V. Bulovic. "Electroluminescence from a Mixed Red-Green-Blue Colloidal Quantum Dot Monolayer," Nano Lett, vol. 7, pp. 2196-2200, July 2007.

[3] J. H. Park, J. Y. Kim, B. D. Chin, Y. C. Kim, J. K. Kim, and O. O. Park "White emission from polymer/quantum dot ternary nanocomposites by incomplete energy transfer," Nanotechnology, vol. 15, pp. 1217-1220, September 2004

[4] C. Y. Huang, T. S. Huang, C. Y. Cheng, Y. C. Chen, C. T. Wan, M. V. M. Rao, and Y. K. Su, "Three-Band White Light-Emitting Diodes Based on Hybridization of Polyfluorene and Colloidal CdSe-ZnS Quantum
Dots," IEEE Photonics Technol. Lett, vol. 22, pp. 2009-2011, March 2010.

[5] P. T. K. Chin, R. A. M. Hikmet, and R. A. J. Janssen, "Energy transfer in hybrid quantum dot light-emitting diodes," J. Appl. Phys, p. 013108, July 2008.

[6] L. Qian, Y. Zheng, J. Xue, and P. H. Holloway, "Stable and efficient quantum-dot light-emitting diodes based on solution-processed multilayer structures", Nat. Photonics, vol. 5, pp. 543-548, September 2011. 